\title{
Insect Sex Determination: A Cascade of Mechanisms
}

Three conditions are essential for evolution: variation, heredity and time. Most animal species transmit genetic information through sexual reproduction in order to meet the first two conditions. The consequence for sexual reproduction is the need for (at least) two sexes with different reproductive roles. Consequently, evolutionary issues arise, such as differences in morphological and behavioral attributes between the sexes, which are reflected in the plethora of animal sex-determining systems. Indeed, a transition to asexual development may occur, sometimes induced by microbial endosymbionts. All these aspects are represented in the insect sex-determining system, which is the focal topic of this issue of Sexual Development.

The first contribution by van Doorn is a theoretical treatise on the evolutionary forces acting on sex determination, including transitions of environmental and genetic sex determination and male and female heterogamety. In the contribution of Bopp, Saccone and Beye, three modules in the insect sexdetermining cascade are derived: the instructive module, the transduction module and the execution module. They argue how the instructive module, as well as the executive module, are primary targets of evolutionary divergence, while the transduction remains conserved. An intriguing example of this is presented by Scott, Pimsler and Tarone, who discuss the difference between the sex determination system of Lucilia cuprina, where sex is determined by a Y-linked male-determining gene (M) and that of Chrysomya rufifacies, in which the maternal genotype involving a dominant factor $(\mathrm{F} / \mathrm{f})$ determines sex. The contribution by Geuverink and Beukeboom shows, by phylogenetic analysis, that the transformer part of the transduction module may be subject to multiple independent losses or recruitments during evolution.

When sex is determined under haplodiploidy, a transition to asexual reproduction may occur, known as thelytoky. This is addressed by Vorburger, who discusses how diploid male production and other constraints may impede or promote the evolution of thelytoky. A special case, the manipulation 
of haplodiploid sex determination by microbial endosymbionts, is discussed by Ma, Vavre and Beukeboom. A newly discovered mechanism behind haplodiploid sex determination is maternal imprinting. Van de Zande and Verhulst describe how maternal imprinting in Nasonia was inferred and confirmed and provide a refinement and addition to the Maternal Effect Genetic Imprinting Sex Determination model. Another example of imprinting in sex determination, that all involve the elimination or inactivation of the paternal chromosomes, is presented by Sanchez. The Lepidopteran sex determination is an example where the transduction module is unique, since thus far no transformer gene has been identified to be involved in the sex-determining cascade. This system is discussed by Nagaraju, Gopinath, Vandana and Shukla in an article dedicated to Dr. Jawaregowda Nagaraju, who unexpectedly passed away during the preparation of the manuscript.

This special issue is concluded by two papers that present some kind of 'follow-up' of the sex determination process. Samson and Rabinow, in a cautionary way, describe the literature on transcriptomic analysis of somatic sexual development in Drosophila melanogaster after the sex-determining cascade. Possible applications of the knowledge gained by investigating insect sex determination are described by Koukidou and Alphey, who present the considerable progress that has been made in understanding how to manipulate insects to develop new synthetic genetics-based tools for the control of pest insects.

We are convinced that this wide palette of reviews is of interest to all who are investigating sexual development, and we thank all contributors for sharing their expertise to compose this overview of insect sex determination.

Eveline C. Verhulst, Louis van de Zande Groningen, January 2014 